\title{
Entrance Effects of Blood Model Casson Fluid in the Concentric Rings with Inner Ring Rotation
}

\author{
Srinivasa Rao Nadiminti, A. Kandasamy
}

\begin{abstract}
This research article analyzes the entrance effects in concentric rings with rotation of inner ring for the blood model Casson non-Newtonian fluid. The investigation is done with the assumption that, the inner ring rotates with a constant velocity along angular direction also the outer ring is at rest. The finite difference technique was applied to find the velocity profiles, variation of pressure in the radial coordinate direction. Calculation has been done for different annular gap values and Casson number. The compared results for different special cases was made and observed to be concordant.
\end{abstract}

keywords: Entrance effect, Blood model Casson Fluid, Finite Difference technique, concentric rings.

\section{INTRODUCTION}

The effects in the entrance zone of concentric rings of laminar non-Newtonian fluid with inner ring rotation having technical applications like, heat exchangers design, turbo machinery and industries of polymer processing. Very often, laminar flow operations provide optimal conditions to maintain a less power pumping proportionally to the rate of heat transfer. Also in the field of nuclear reactors, this is happening when the cooling rates reduced. All the fluids which having practical applications are not obey Newtonian equation, so they are called as rheological fluids. Various suspensions such as coal, blood, food, paintings, polymer solutions. Here the blood model Casson fluid is taken, which belongs to the fluid class of flow with independent of time.

The Newtonian fluid flow in the entrance zone problem in a concentric rings was studied by [4]. [8] analyzed the entrance effects of Bingham fluids in the concentric rings and analyzed the boundary layer thickness. [2] studied the relationship for the Casson fluid with outer ring rotation and inner ring is at rest between two rotating cylinders in the annular space. [11]used finite difference technique to analyze the non-Newtonian fluid in the geometry of concentric rings with the previous mentioned conditions. The relation of stress and strain for the Casson fluid is given by Fung [6]

Revised Manuscript Received on December 15, 2019

Srinivasa Rao Nadiminti, Division of Mathematics, Department of Science and Humanities Vignan's Foundation for Science, Technology and Research (VFSTR) Vadlamudi, Guntur, Andhrapradesh - 522213, India. email: srinudm@gmail.com

A.Kandasamy, Department of Mathematical and Computational Sciences National Institute of Technology Karnataka, Mangalore, Karnataka-575025, India. e-mail: kandy@ nitk.ac.in

$$
\tau^{\frac{1}{2}}=\tau_{0}^{\frac{1}{2}}+K c \gamma^{\frac{1}{2}}
$$

Here shear stress is denoted by $\tau, \gamma$ is strain rate. Yield value and Casson's viscosity denoted by $\tau_{0}$ and $K_{c}{ }^{2}$. This constitutive equation was successfully applicable to chocolate and blood. Further, Casson fluid flow with a side branch in a narrow tube has been investigated by [9]. Homogeneous porous medium for the flow of Casson fluid in a pipe has been considered by [5]. [1] investigated Magneto hydrodynamic flow with heat transfer for the nonNewtonian fluid in an eccentric rings. Analytical solution in the entrance region blood flow in a concentric annuli has been obtained by [3] assuming the blood to obey Casson model. Recently [7,10] analyzed the entrance effects of non-Newtonian flow in the concentric rings.

The problem of Casson fluid in the concentric rings to the flow of the inlet region has been studied in the present work. The analysis was performed by taking the nonrotating outer ring is at rest and rotating the inner ring. Using the Prandtls boundary layer hypotheses, conservation equations of momentum, mass are solved and analyzed by finite difference technique. The algebraic system of equations in nonlinear form are obtained and was solved by the iterative method of Newton-Raphson. The compared results for different special cases was made and observed to be concordant.

\section{PROBLEM FORMULATION}

Fig.1 shows the geometrical Representation of the problem. The Casson fluid entered in to the concentric rings horizontally with $\mathrm{R} 1$ and $\mathrm{R} 2$ are the inner and outer radius respectively, with the uniform uniform velocity $\mathrm{u} 0$ along the axial direction with $\mathrm{p} 0$ as the initial pressure. The non rotating outer ring is at rest and the inner ring rotating with the angular velocity. The flow is axisymmetric, incompressible and laminar. The system of cylindrical polar coordinates apt to the geometry of the problem for the Casson fluid in the entrance are given by

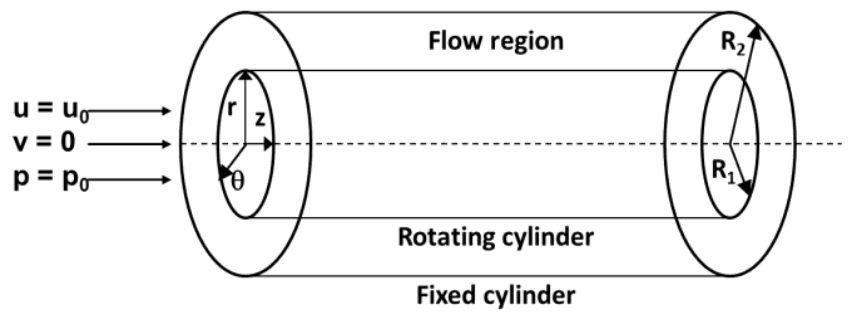

Fig.1: Geometrical Representation to the Physical Problem 
Equation of continuity: $\frac{\partial(r v)}{\partial r}+\frac{\partial(r u)}{\partial z}=0$

Z -momentum

equation: $\frac{\partial u}{\partial r} v+\frac{\partial u}{\partial z} u=\frac{1}{\rho} \frac{\partial p}{\partial z}+\frac{1}{\rho r} \frac{\partial}{\partial r}\left(r\left(\tau_{0}+K^{2}{ }_{c} \frac{\partial u}{\partial r}+\right.\right.$

$2 K c \tau O \partial u \partial r$

$\theta$-momentum equation:

$\frac{\partial w}{\partial r} v+\frac{\partial w}{\partial z} u+\frac{v w}{r}=\frac{1}{\rho r^{2}} \frac{\partial}{\partial r}\left(r^{2}\left(\tau_{0}+K^{2}{ }_{c} \frac{\partial}{\partial r}\left(\frac{w}{r}\right)+\right.\right.$

$2 \operatorname{Kc\tau } 0 \partial \partial r(w r)$

R-momentum equation: $\frac{w^{2}}{r}=\frac{1}{\rho} \frac{\partial p}{\partial r}$

Here the velocities in the direction $\mathrm{z}, \mathrm{r}, \theta$ represented by $\mathrm{u}, \mathrm{v}, \mathrm{w}$, temperature of the fluid is denoted by $\mathrm{t}, \rho$ is the fluid density, pressure of the fluid is denoted by $\mathrm{p}$.

Following are the hydrodynamic part boundary conditions

$$
\begin{aligned}
& \text { at } \mathrm{z}=0, \mathrm{p}=\mathrm{p}_{0} \\
& \qquad \begin{array}{l}
\text { at } \mathrm{z}=0, \mathrm{R}_{1}<\mathrm{r}<\mathrm{R}_{2}, \mathrm{u}= \\
\text { with } \mathrm{z}>0, \mathrm{r}=\mathrm{R}_{2} \quad, \mathrm{v}=\mathrm{u} \\
\text { with } \mathrm{z}>0 ; \mathrm{r}=\mathrm{R}_{1} \quad, \mathrm{v}=\mathrm{u}
\end{array}
\end{aligned}
$$

Continuity equation (2) with the boundary conditions (6) can be expressed as

$\int_{R 1}^{R 2} 2 r u d r=\left(R 2^{2}-R 1^{2}\right) u 0$

Consider the following dimensionless equations

$R=\frac{r}{R 2}, U=\frac{u}{u 0}, V=\frac{\rho v R 2}{K c^{2}}, W=\frac{w}{\omega R 1}, N=\frac{R 1}{R 2}, P=\frac{p-p 0}{\rho u 0^{2}}$

$Z=\frac{2 z(1-N)}{R 2 R e}, Y_{C}=\frac{\tau 0_{R 2}}{u 0 K c^{2}}$

$T_{a}=\frac{2 \omega^{2} \rho^{2} R 1^{2}(R 2-R 1)}{\mu r^{2}(R 1+R 2)}$, where $\mu r=K c^{2}\left(\frac{\omega R 1}{R 2}\right), R e=$ $\frac{2 R 2(1-N) \rho u 0}{K c^{2}}$

Here Yc is the Casson value, Ta Taylors number, Re Reynolds number and $\mathrm{N}$ is the annular gap of the annuli. Dimensionless equations of (2)-(5) and (7) are the following

$\frac{\partial W}{\partial R} V+\frac{\partial W}{\partial Z} U+\frac{V W}{R}=\frac{4 Y c^{\frac{1}{2}}}{R}\left(\frac{\partial W}{\partial R}-\frac{W}{R}\right)^{\frac{1}{2}}+Y C^{\frac{1}{2}}\left(\frac{\partial W}{\partial R}-\right.$ $W R-12 \partial 2 W \partial R 2-1 R \partial W \partial R+W R 2+\partial 2 W \partial R 2+1 R \partial W \partial R$ $+W R 2+2 Y C R$

$\frac{\partial U}{\partial R} V+\frac{\partial U}{\partial Z} U=$

$-\frac{\partial P}{\partial Z}+\frac{1}{R} \frac{\partial U}{\partial R} \frac{2 Y c^{\frac{1}{2}}}{R}\left(\frac{\partial U}{\partial R}\right)^{\frac{1}{2}}+Y C^{\frac{1}{2}}\left(\frac{\partial W}{\partial R}\right)^{-\frac{1}{2}}\left(\frac{\partial^{2} U}{\partial R^{2}}\right)+\frac{\partial^{2} U}{\partial R^{2}}+\frac{Y C}{R}$

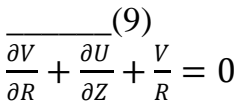

$\frac{R e^{2}(1-N)}{2(1+N) T a} \frac{\partial P}{\partial R}=\frac{W^{2}}{R}$

Also $\int_{N}^{1} 2 R U d R=\left(1-N^{2}\right)$

The dimensionless conditions for the hydrodynamic part associated with the boundary conditions (6) are given by at $\mathrm{Z}=0, \mathrm{~N}<\mathrm{R}<1, \mathrm{U}=1$

at $\mathrm{Z}=0, \mathrm{P}=0$

with $\mathrm{Z}>0, \mathrm{R}=\mathrm{N}, \mathrm{V}=\mathrm{U}=0 ; \mathrm{W}=1$

with $\mathrm{Z}>0, \mathrm{R}=1, \mathrm{~V}=\mathrm{U}=\mathrm{W}=0$

\section{Numerical solution}

We adopted and considered the numerical method from the work of [4]. Fig.2 show the mesh network for the given problem. The radial and axial direction grid sizes are represented by $\Delta R$ and $\Delta Z$ respectively.

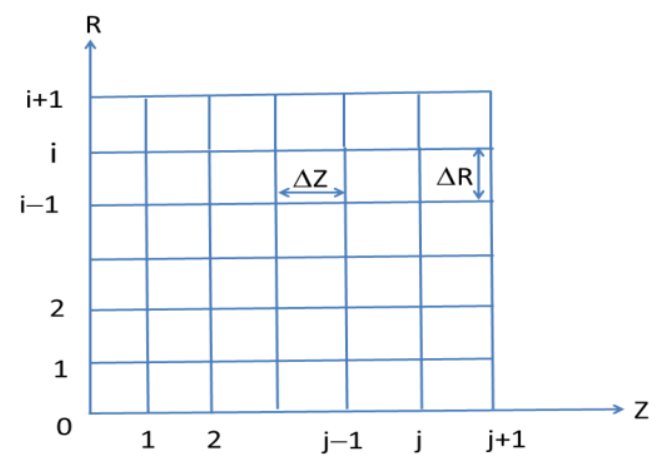

Fig.2: Grid representation for the problem

The solution of finite difference equation of (8)-(12) by the iterative procedure, using the previous mentioned Procedure up to the flow becomes developed fully in the both axial and tangential directions.

\section{EXPERIMENTAL STUDY}

The finite difference analysis have been obtained for different values of Casson Number Yc , annular gap values $\mathrm{N}$ and various parameters. Here, the velocity profiles and pressure of the annuli have been shown in following figures.

Figure 3-5 show the velocity profiles in the tangential direction with aspect ration values 0.8 and 0.3 and for different values of Casson numbers Yc. The results has been done for various values of the parameter Rt to study the effect of rotation of inner cylinder. The values corresponding to $\mathrm{Rt}=1$ and 20 are depicted in the figures. The tangential velocity values are low from the inner ring to the non-rotating outer ring of the annuli. Also it is observed that with the high of annular gap, the tangential velocity profiles are also high. 
Figure 6-8 show the velocity profiles in the axial direction with aspect ration values 0.8 and 0.3 at axial positions of $\mathrm{Z}$ $=0.01,0.03$ and for various values of the Casson numbers Yc . Also observed that, with increment of the aspect ratio, the axial velocities are high at all values of Casson numbers. Then it is found, the velocity profile looks the parabolic shape with Casson number Yc being zero (Newtonian fluid).

The velocity profiles along the radial direction with aspect ration values 0.8 and 0.3 for various values of the Casson numbers $\mathrm{Yc}$ at different axial positions $\mathrm{Z}$ are shown in Figure 9-11. Again, the parameter Rt values are taken as 1 and 20 for computational purpose. At the region near the non-rotating outer ring the radial velocity values are negative, because it is in the radial coordinate opposite direction. Near the rotating inner ring, it has the positive values since it is in same radial coordinate direction. Values of the radial distribution is decreases with increase of Casson number Yc. Because of the angular rotation of the inner ring of the annulus this phenomena is observed.

Figure 12-14 show the pressure variation in the radial direction $\mathrm{R}$ with aspect ration values 0.8 and 0.3 for different values of Casson numbers Yc. It is observed that the values of $\mathrm{P}$ going from the low at the rotating ring to a high at the non-rotating outer ring of the annulus. Also, it is observed that increment in the value of Casson numbers $\mathrm{Y}$ c , decrease the pressure values P. Further, it is noted that near the outer wall region the pressure is not changed.

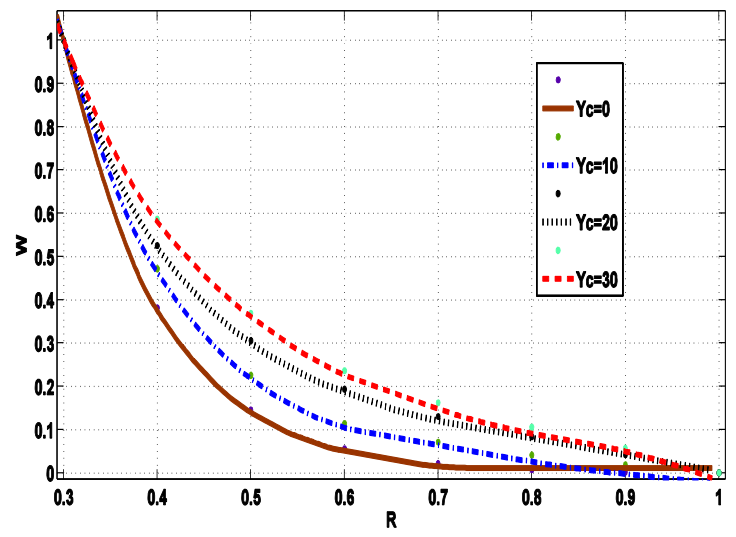

Fig.3: Distribution of tangential velocity with annular gap 0.3

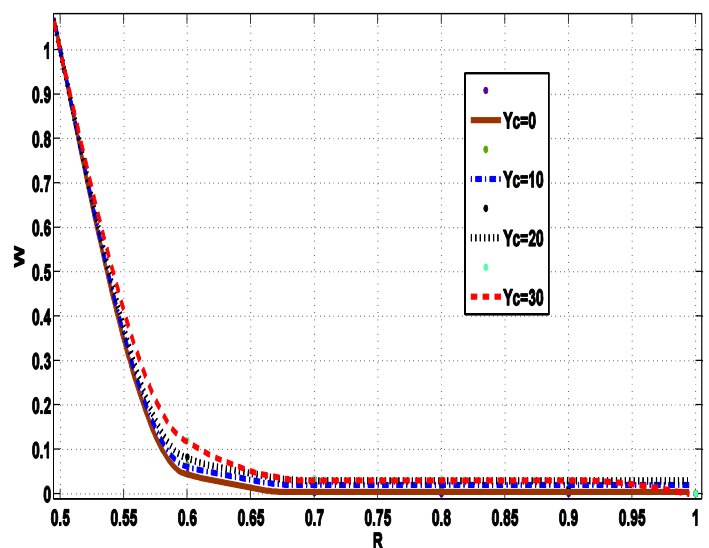

Fig.4: Distribution of tangential velocity with annular gap 0.5

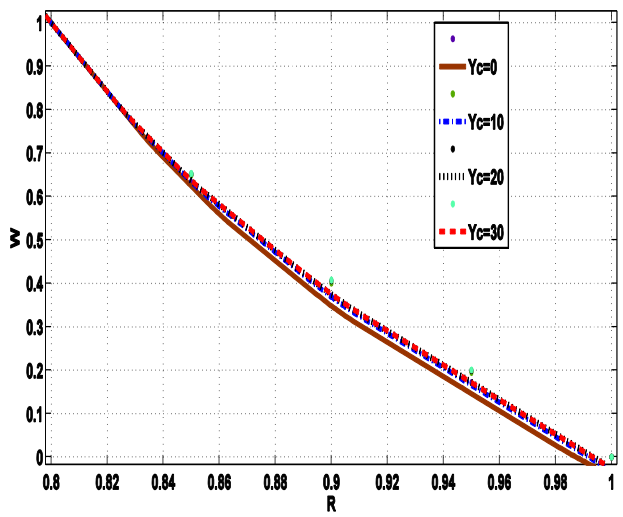

Fig.5: Distribution of tangential velocity with annular gap 0.8

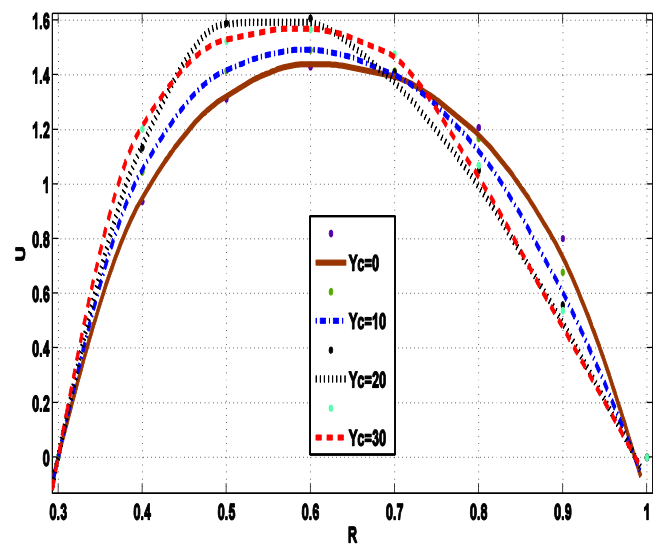

Fig.6: Distribution of axial velocity with annular gap 0.3 


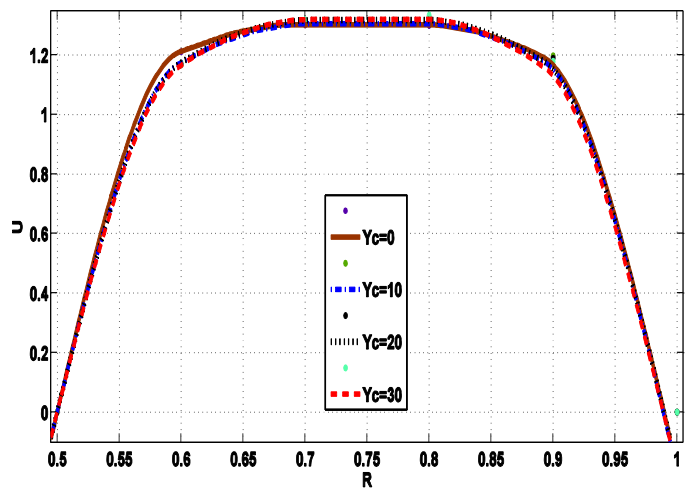

Fig.7 Distribution of axial velocity with annular gap 0.5

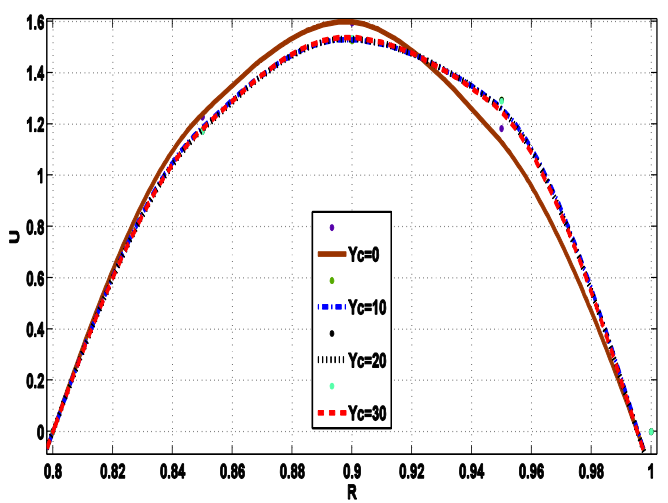

Fig.8 Distribution of axial velocity with annular gap 0.3

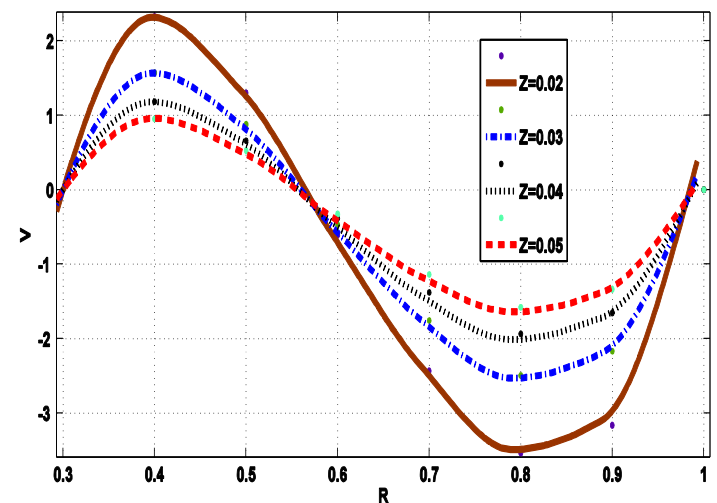

Fig.9 Distribution of radial velocity with annular gap 0.3

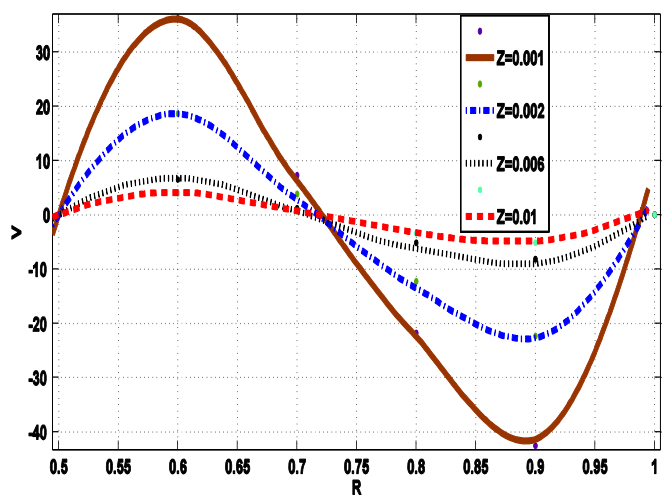

Fig.10 Distribution of radial velocity with annular gap 0.5

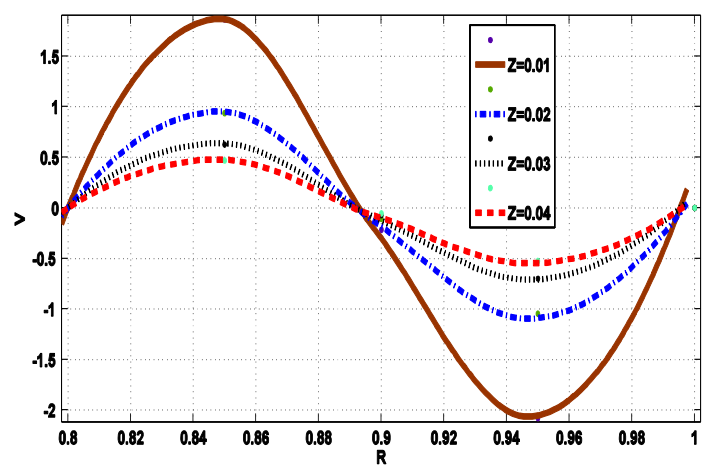

Fig.11 Distribution of radial velocity with annular gap 0.8

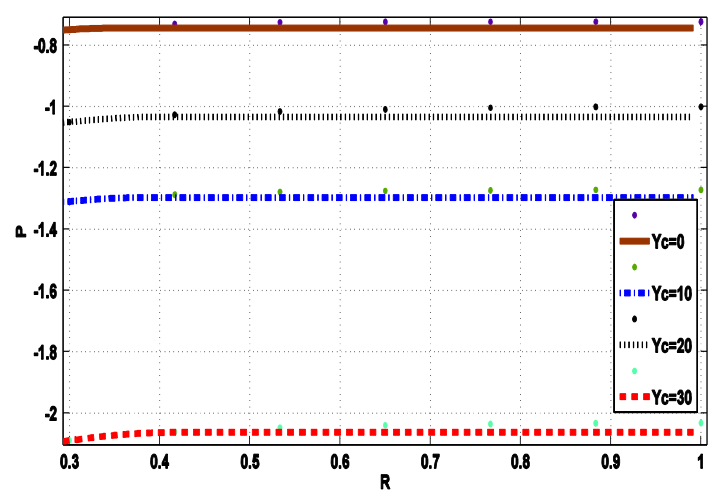

Fig.12 Distribution of pressure with annular gap 0.3 


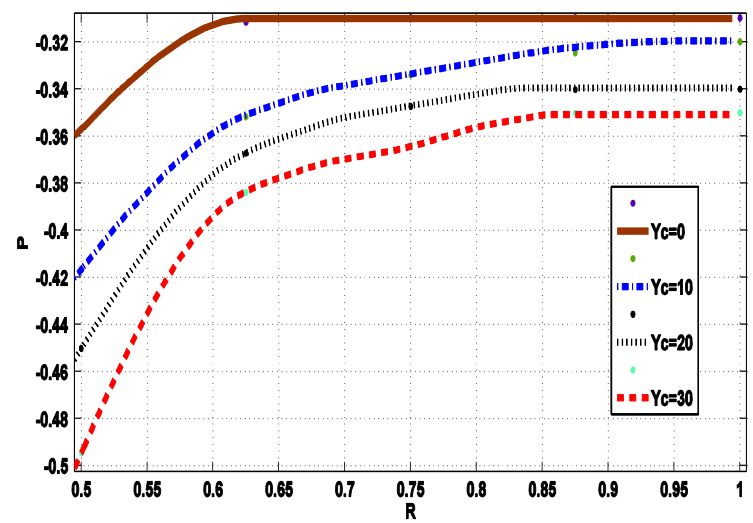

Fig.13 Distribution of pressure with annular gap 0.5

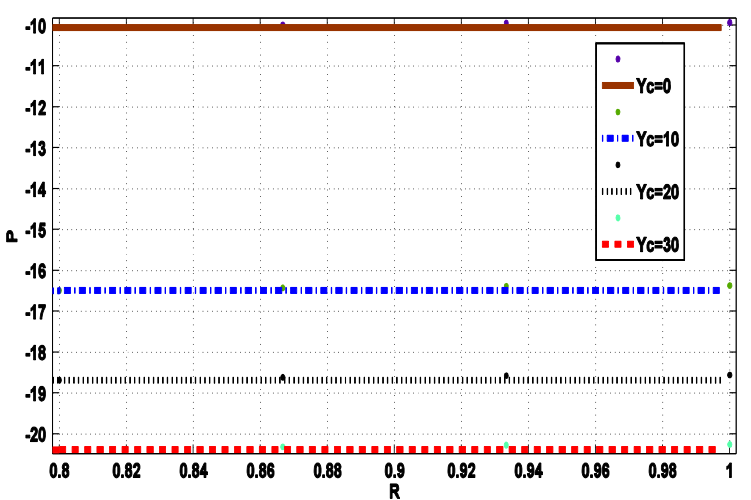

Fig.14 Distribution of pressure with annular gap 0.8

\section{CONCLUSION}

Here we presented the numerical solution to the blood model casson fluid in entrance zone of concentric annuli. The results were estimated for all the values of the Casson number and the aspect ratio. The graphs of the velocities and pressure in the radial coordinate direction has been geometrically represented and the comparing of the current analysis with the available results for special cases was made and observed to be concordant.

From the above analysis, the following points can be drawn:

1. From the above figures we can say that, from the inner ring to the non rotating outer ring of the annuli, the tangential profiles are decreases.

2. With a fixed Casson number $\mathrm{Y} \mathrm{c}$, the velocity $\mathrm{U}$ increases along the axial direction, if we decrease the annular gap.

3. The pressure is found to be low at the rotating wall and gradually increasing to a high at the non-rotating wall for all values of Casson numbers. and pressure variation is not much considered along the radial direction near the nonrotating wall of the annulus.

4. As observed in the case of Bingham study here also decreases the temperature from the inner ring to the nonrotating outer ring.

\section{REFERENCES}

1. Ahmed, M.E.S., Attia, H.A., Magneto hydrodynamic flow and heat transfer of non-Newtonian fluid in an eccentric annulus. Canadian Journal of Physics, 76, 391-401 (1998)

2. Batra, R.L and Bigyani Das, Flow of a Casson Fluid between Two Rotating Cylinders. Fluid Dynamic Research. 9, 133-141 (1992)

3. Batra, R. L. and Bigyani Jena, Entrance region flow of blood in concentric annulus. International Journal of Engineering Science. 28, 407-419 ( 1990)

4. Coney, J. E. R and El-Shaarawi, M. A. I., A contribution to the numerical solution of developing laminar flow in the entrance region of concentric annuli with rotating inner walls. ASME Trans. Journal of Fluid Engineering. 96, 333-340(1974)

5. Dash, R.K., Mehta, K.N., \& Jayaram, G., Casson fluid flow in a pipe filled with a homogeneous porous medium. International Journal of Engineering Science, 34, 1145-1156 (1996)

6. Fung, Y.C., Biomechanics: Mechanical Properties of Living Tissues. Springer, New York (1981)

7. Kandasamy, A., Srinivasa Rao Nadiminti, Entrance region flow in concentric annuli with rotating inner wall for HerschelBulkley fluids. International Journal of Applied and Computational Mathematics, 1-2, 235-249 (2015)

8. Mishra, I.M., Surendra Kumar, Entrance Region Flow of Bingham Plastic Fluids in Concentric Annulus. Indian Journal of Technology, 23, 81-87 (1985)

9. Misra, J.C., Ghosh, S.K., Flow of a Casson fluid in a narrow tube with a side branch. International Journal of Engineering science, 38, 2045-2077 (2000)

10. Kandasamy, A., Srinivasa Rao Nadiminti, Entrance region flow in concentric annuli with rotating inner wall for Bingham fluids. Journal of Computational and Applied Mechanics, 11-2, 137-157 (2016)

11. Sayed-Ahmed, M.E., Hazem Sharaf-El-Din, Entrance region flow of a power-law fluid in concentric annuli with rotating inner wall. International Communications in Heat and Mass Transfer, 33, 654$665(2006)$ 